\title{
Synergistic synthesis of quasi-monocrystal CdS nanoboxes with high-energy facets
}

\author{
Li-Li Han ${ }^{\dagger}, \|$, Sergei A. Kulinich ${ }^{\ddagger} \S$, Yang-Yang Zhang ${ }^{\dagger}$, Jin Zou ${ }^{\perp}$, Hui Liu\#, Wei-Hua Wang ${ }^{\nabla}$, Hui Liu ${ }^{\nabla}$, \\ Hao-Bo Li ${ }^{\nabla}$, Jing Yang ${ }^{\dagger}$, Huolin L. Xin", ShiZhang Qiao ${ }^{*, \dagger} \circ \&$ Xi-Wen Du*, ${ }^{*}$ \\ ${ }^{\dagger}$ School of Materials Science and Engineering, Tianjin University, Tianjin 300072, China \\ ${ }^{\dagger}$ Institute of Innovative Science and Technology, Tokai University, Hiratsuka, Kanagawa 259-1292, Japan \\ ${ }^{\S}$ School of Engineering and Applied Science, Aston University, Birmingham, B4 7ET, UK \\ "Center for Functional Nanomaterials, Brookhaven National Laboratory, New York 11973, USA \\ ${ }^{\perp}$ Materials Engineering and Centre for Microscopy and Microanalysis, The University of Queensland, QLD 4072, Australia \\ ${ }^{\#}$ School of Material Science and Engineering, Hebei University of Technology, Tianjin 300130, China \\ ${ }^{\nabla}$ Department of Electronics, College of Electronic Information and Optical Engineering, Nankai University, Tianjin 300071, \\ China
}

${ }^{\circ}$ School of Chemical Engineering, The University of Adelaide, SA 5005, Australia

*Corresponding author: xwdu@tju.edu.cn, s.qiao@adelaide.edu.au

\begin{abstract}
Hollow nanostructures with highly-oriented-lattice structure and active facets are promising for catalytic applications, while their preparation via traditional approaches requires multiple steps and time- and energy-consuming. Here, we demonstrate a new one-step strategy involving two complementary reactions, which promote each other, that is capable of producing unique hollow nanoparticles. Specifically, we apply synergic cooperation of cation exchange and chemical etching to attack PbS nanosized cubes and produce $\mathrm{CdS}$ quasi-monocrystal nanoboxes which possess the smallest dimensions reported so far, metastable zinc-blende phase, large specific surface area, and particularly high-energy $\{100\}$ facets directly visualized by aberration-corrected scanning transmission electron microscopy. These properties, in combination, allow the nanoboxes to acquire exceptional photocatalytic activities. As an extension of the approach, we use the same strategy to prepare $\mathrm{Co}_{9} \mathrm{~S}_{8}$ and $\mathrm{Cu}_{7.2} \mathrm{~S}_{4}$ single-crystal hollow nanooctahedrons. Hence, the proposed approach based on synergic reactions exhibits great potential in engineering unique nanostructures with superior properties.
\end{abstract}

\section{INTRODUCTION}

Hollow nanostructures possess large specific area, unique exposed crystal planes and devisable energy levels, which makes them highly attractive for applications in various fields related to catalysis, energy storage and conversion, biomedicine, etc ${ }^{1,2}$. So far, the most common synthetic routes to prepare hollow nanostructures typically involved multiple steps. For example, the template-based synthesis of hollow nanostructures is usually comprised of three steps: (i) template preparation, (ii) shell growth on its surface, and (iii) template elimination ${ }^{3,4}$. Typically, such multi-step processes are time- and energy-consuming. An alternative way is to combine two or more processes and run them simultaneously in one system. Such a synthetic route, hereafter named "synchronous approach", has succeeded in the synthesis of nanocavities ${ }^{5}$, nanocages ${ }^{6,7}$, nanoframes ${ }^{2}$, nanotubes ${ }^{8,9}$, complex 3-dimensional colloidal nanoparticles ${ }^{10}$, and yolk-shell nanostructures ${ }^{4}$. Nevertheless, the component reactions used in most synchronous approaches typically work separately rather than complement each other. As a result, their products often have a relatively low quality with polycrystallinity, non-uniform shell thickness and damaged shells $5,11,12$. Moreover, only a few works on the preparation of non-spherical hollow particles were reported because of the structural instability of such structures ${ }^{11,13,14}$.

It is anticipated that the synchronous approaches can be further improved by carefully selecting complementary component reactions, so that they can promote each other to engineer complex nanostructures with superior properties. This strategy is defined as "synergistic reactions" to distinguish it from the commonly used synchronous reactions. It is noted that, on one hand, the cation exchange is well-known as a powerful approach for synthesizing nanostructures through the cation substitution within the anion frame, in which the product can generally preserve the crystal structure of the templates, even at the nanoscale ${ }^{15,16}$. On the other hand, the chemical etching is a common practice to remove the template or inner material and produce hollow nanostructures ${ }^{4}$. When these two reactions collaboratively attack single-crystal nanoparticles, chemical etching may promote the cation exchange by creating new exchange sites and decreasing the diffusion length to prepare hollow structured nanoparticles, which is different from the multiplestep reactions or synchronous approach involving anion exchange and the Kirkendall effect ${ }^{7,17-20}$. Thus far, there has not been any report about the synthesis of hollow nanoparticles using this strategy.

Cadmium sulfide (CdS), an important semiconductor with a band gap of $2.4 \mathrm{eV}$ at room temperature, has attracted considerable interest in photocatalysis ${ }^{21,22}$, light-emitting diodes ${ }^{23}$, solar cells $^{24}$, and other optoelectronic applications ${ }^{25}$. Many synthesis methods, such as electrochemical deposition ${ }^{26}$, solvothermal (hydrothermal) synthesis ${ }^{22,27}$, chemical vapor deposition $(\mathrm{CVD})^{28}$ and microwave irradiation ${ }^{29}$, have been developed to prepare $\mathrm{CdS}$ nanostructures, among which are irregular-shaped nanoparticles $^{21,30}$, nanotubes ${ }^{31}$, nanowires ${ }^{22}$, nanorods ${ }^{32}$, nanobelts $^{33}$, and so on. Nevertheless, the reported CdS catalysts usu- 
ally expose low-energy facets due to surface energy minimization during the crystal-growth processes ${ }^{34-36}$, which limits their catalytic performance ${ }^{37,38}$. Thus, so far, there has not been any general approach for the synthesis of cubic CdS with ultrafine size, highly-active surfaces and large specific surface area for advanced photocatalytic applications.

In the present work, applying the synergic reactions of chemical etching and cation exchange to $\mathrm{PbS}$ nanocubes (NCs), we for the first time obtained the smallest ever reported $\mathrm{CdS}$ quasimonocrystal nanoboxes (QMNBs) (14 nm in size and with 2.5$\mathrm{nm}$-thick walls) with uniform sizes and uncommon cubic structure. Aberration-corrected scanning transmission electron microscopy (STEM) shows that the prepared CdS QMNBs are primarily terminated with $\{100\}$ facets. The density function theory (DFT) calculations suggest that, among various low-index planes of the zinc-blende CdS phase, the $\{100\}$ planes own the highest surface energy (even an order of magnitude larger than that of the $\{110\}$ planes). The CdS QMNBs with high energy $\{100\}$ facets demonstrate superior photocatalytic efficiency over CdS nanoparticles (NPs) with the same quantity but even larger specific surface area, which makes the new material very promising for catalytic applications. The new strategy is further extended successfully to prepare $\mathrm{Co}_{9} \mathrm{~S}_{8}$ and $\mathrm{Cu}_{7.2} \mathrm{~S}_{4}$ single-crystal hollow nanooctahedrons (SCHNOs), demonstrating it is a promising method to develop new hollow nanostructures.

\section{- EXPERIMENTAL SECTION}

Materials synthesis. The preparation approach is depicted in Scheme 1 and the details are given in Supporting Information. The 15.6-nm-sized PbS NCs were obtained by a hot-injection method. The procedures were mainly same as previously reported in the literature with only slight modifications ${ }^{39} .54 \mathrm{mg}$ of sulfur powder dissolved in $5 \mathrm{~mL}$ of oleylamine (OAm) was injected into a three-neck flask with $0.56 \mathrm{~g}$ of $\mathrm{PbCl}_{2}$ dissolved in $10 \mathrm{~mL}$ of OAm at $220{ }^{\circ} \mathrm{C}$. The mixture was aged for $1 \mathrm{~h}$, resulting in a black colloidal solution. After cooling down, the black solution was centrifugated, washed with ethanol and finally dried in a vacuum desiccator. $\mathrm{CdS}$ nanoboxes were prepared according to the following procedures. First, $0.2658 \mathrm{~g}$ of $\mathrm{PbS}$ NCs was dispersed in $100 \mathrm{~mL}$ of oleic acid (OA) to obtain a suspension. Then, $3 \mathrm{~mL}$ of the suspension was mixed with 15 $\mathrm{mL}$ of $0.5 \mathrm{M}$ cadmium acetate dissolved in OA. After the reaction proceeded at $180{ }^{\circ} \mathrm{C}$ for a given period of time $(2 \sim 35 \mathrm{~min})$ in a three-neck flask, the mixture was momentarily cooled down to room temperature and centrifugated to separate the deposit. Finally, the product was washed with ethanol several times prior to further characterization. The preparation procedures for $\mathrm{Co}_{9} \mathrm{~S}_{8}$ and $\mathrm{Cu}_{7.2} \mathrm{~S}_{4}$ SCHNOs were same as described above for the CdS QMNBs except for that $0.05 \mathrm{M} \mathrm{Co}$ (II) acetate tetrahydrate and $0.05 \mathrm{M} \mathrm{Cu}$ (II) acetate monohydrate were used instead of $0.5 \mathrm{M} \mathrm{Cd}$ acetate trihydrate and the reaction temperatures were set at $300{ }^{\circ} \mathrm{C}$ and $150{ }^{\circ} \mathrm{C}$, respectively. (a)

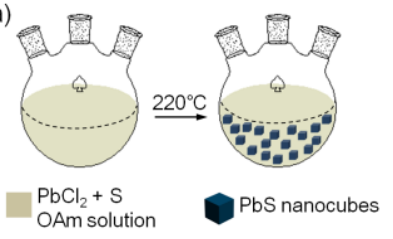

(b)

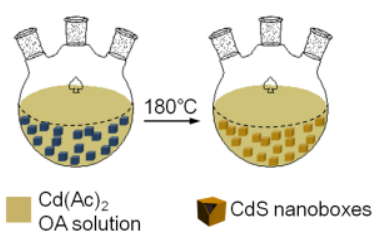

Scheme 1. Schematic illustration of (a) synthesis of $\mathrm{PbS}$ nanocube templates and (b) one-step transformation into $\mathrm{CdS}$ nanoboxes.

Characterization of materials. Phase analysis was carried out by X-ray diffractometry (XRD, Bruke D8 advance instrument). The product morphology was determined by transmission electron microscopy (TEM, FEI Technai G2 F20, equipped with a field emission gun operating at $200 \mathrm{kV}$ ). The composition was analyzed with an Oxford INCA energy-dispersive spectroscopy (EDS) module attached to the TEM microscope. Atomic-resolution annular dark-field (ADF) and annular brightfield $(\mathrm{ABF})$ images were obtained with an Enfina spectrometer on a Hitachi 2700C STEM. A Hitachi S-4800 SEM was used to analyze the NP shape and surfaces. The light absorption spectra of the samples were examined using a Hitachi 3010 UV-vis absorption spectrometer. A solar simulator (Sciencetech, SS150) was used as the light source for photocatalysis experiments. Nitrogen sorption measurements were performed at $77 \mathrm{~K}$ using an autosorbiQ instrument (Quantachrome, US).

Surface energy calculations of zinc-blende CdS. The DFT calculations of the surface energies were performed using the Vienna $a b$ initio simulation package (VASP) with choosing the generalized-gradient approximation (GGA) of Perdew, Burke and Ernzerhof (PBE) for the exchange-correlation function ${ }^{40}$. The Monkhorst-Pack k-point grids of $(8 \times 8 \times 8)$ and $(8 \times 8 \times 1)$ were adopted for the bulk and slab calculations, respectively. All the neighbor slabs were separated by a $15-\AA$ vacuum space. In the geometry optimizations, the positions of all the atoms were fully relaxed until the force on each atom converged to $0.01 \mathrm{eV} / \AA \AA$. For the bulk CdS, the optimized lattice constant was $5.832 \AA$, which agreed well with the experimental vales of $5.795 \AA$ or $5.830 \AA^{41}$. For the (100), (110) and (111) slab models, each slab contained 16 layers with $16 \mathrm{Cd}$ and $16 \mathrm{~S}$ atoms. The two top layers and two bottom layers were fully relaxed while the other layers were fixed.

The surface energies were calculated using the following formula,

$$
E_{\text {surface }}=1 / 2\left[E_{\text {slab }}-n E_{\text {bulk }}\right] / A
$$

where $A$ is the surface area of the slab model, $E_{\text {bulk }}$ is the bulk energy per unit cell, and $\mathrm{E}_{\text {slab }}$ is the energy of each slab model, which contains $n$ bulk unit cells. The constant $1 / 2$ indicates that two surfaces of the slab model are taken into account.

\section{- RESULTS AND DISCUSSION}

The $\mathrm{PbS}$ NCs used as templates exhibit a uniform cubic morphology and narrow size distribution centered around $15.6 \mathrm{~nm}$ (Figure 1a). Upon reacting with OA-dissolved $\mathrm{Cd}(\mathrm{Ac})_{2}$ at $180^{\circ} \mathrm{C}$, the morphology of the PbS NCs changes with time. After $10 \mathrm{~min}$, a light contrast preferably appears at the vertexes and edges of the NCs, and tiny holes can be found on the surfaces of some PbS NCs (Figure 1b), indicating etching initiates locally rather than evenly over the entire surface. The small holes grow bigger as the reaction time increases to $20 \mathrm{~min}$ (Figure 1c). The interior of PbS NCs is almost etched 


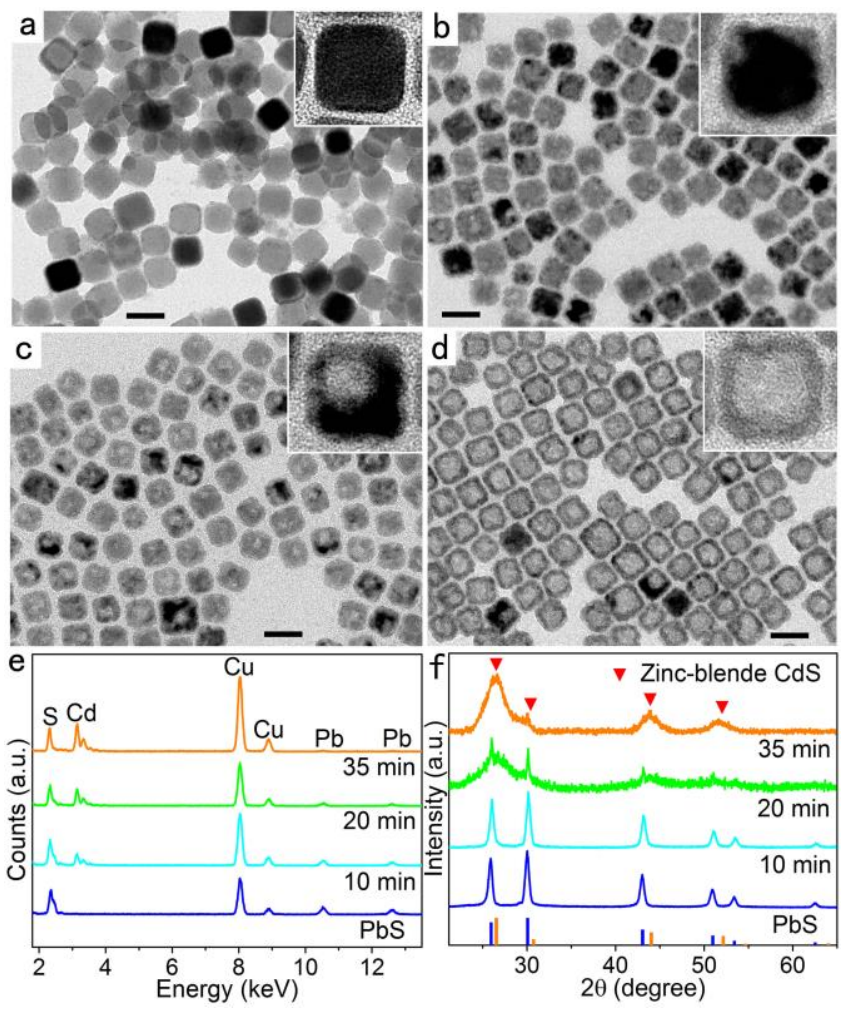

Figure 1. Evolution from $\mathrm{PbS}$ nanocubes to $\mathrm{CdS}$ nanoboxes. TEM images of the products obtained at $180{ }^{\circ} \mathrm{C}$ after (a) $0 \mathrm{~min}$, (b) $10 \mathrm{~min}$, (c) $20 \mathrm{~min}$ and (d) $35 \mathrm{~min}$ reaction. The scale bar is $20 \mathrm{~nm}$. (e) and (f) are EDS spectra and XRD patterns, respectively, of the products after different reaction times.

away completely when the reaction reaches $35 \mathrm{~min}$, and most of the solid $\mathrm{PbS}$ NCs are found to be transformed into well-defined nanoboxes. The QMNBs have a mean outer size of $\sim 14$ $\mathrm{nm}$ with a wall thickness of $2.5 \mathrm{~nm}$ (Figure 1d), thus being the smallest compound nanoboxes ever reported. Their specific surface area, $63 \mathrm{~m}^{2} \mathrm{~g}^{-1}$, estimated from the Brunauer, Emmett and Teller (BET) measurement, is by far larger than that of PbS NCs $\left(0.76 \mathrm{~m}^{2} \mathrm{~g}^{-1}\right)$ (Figure $\left.\mathrm{S} 1\right)$. The composition and structure of the $\mathrm{PbS}$ NC templates over reaction duration were investigated by means of EDS and XRD. As clearly seen in Figures 1e and 1f, $\mathrm{PbS}$ peaks gradually decrease while those of $\mathrm{CdS}$ increase with reaction time. When the reaction time reaches $35 \mathrm{~min}, \mathrm{PbS}$ almost disappears, and the main product is the zinc-blende structured CdS.

The atomic surface structure of the obtained CdS nanoboxes is revealed by aberration-corrected STEM. As shown in Figure $2 \mathrm{a}$, the product particle is a quasi-monocrystal nanobox (QMNB) with well oriented lattices
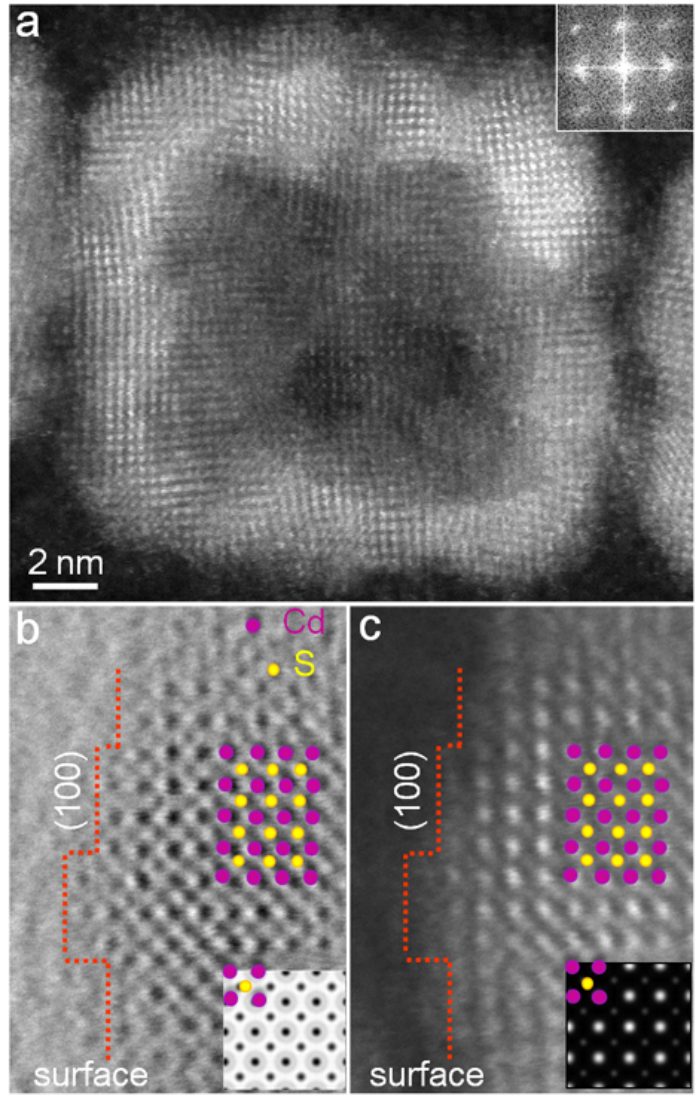

Figure 2. Revealing atomic surface structure of $\mathrm{CdS}$ QMNBs. (a) Atomic-resolution ADF-STEM image of one QMNB. The inset is a corresponding FFT pattern. (b) Atomicresolution ABF-STEM and (c) atomic-resolution ULAADFSTEM images of surface structure of CdS QMNB along the [001] zone axis after Richardson-Lucy deconvolution. The exposed facet profiles are outlined by red dotted lines. The insets show ABF and ULAADF simulations, respectively. The sulfur columns can be directly visualized in the ABF-STEM and ULAADF-STEM images.

and a few small-angle grain boundaries, which is also shown in the high resolution TEM (HRTEM) image in Figure S2. Due to the relatively low atomic number of sulfur compared to cadmium, sulfur columns cannot be directly imaged by the traditional annular dark-field STEM imaging. To visualize the sulfur columns, we employed two new optical geometries, i.e. the annular bright-field (ABF) and the ultra-low-angle annular darkfield (ULAADF) STEM image. The simultaneously acquired atomic-resolution images taken along the [001] zone axis (Figures $2 \mathrm{~b}$ and $2 \mathrm{c}$ ) clearly depict sulfur and cadmium positions on the surface of the CdS QMNB. From the images, the projected zinc-blende unit cell is composed of a periodic square array of Cd columns surrounded by $\mathrm{S}$ columns, which is consistent with $\mathrm{ABF}$ and ULAADF simulations (insets of Figures $2 \mathrm{~b}$ and $2 \mathrm{c}$ ). The $\{100\}$-determined facets and small steps on the surface are observed in Figures $2 \mathrm{~b}$ and $2 \mathrm{c}$. It should be noted that the observed $\{100\}$ surface is Cd-terminated, and as such it can be stabilized by oleic acid molecules ${ }^{42}$. 
To obtain CdS nanoboxes with larger $\{100\}$ facets, we then employed 50-nm-sized PbS NCs as the template. The morphology evolution of the products was found to follow the same mode as that for 15.6-nm-sized PbS NCs according to the TEM and TEM observations (Figure S3), and QMNBs with a shell thickness of $2.7 \mathrm{~nm}$ were produced at $180{ }^{\circ} \mathrm{C}$ (Figure S5a). Moreover, elemental mapping and line-scan were performed to reveal the elemental distribution in the intermediate product obtained after $10 \mathrm{~min}$ of reaction (its morphology is presented in Figures S3c and S3d). The results shown in Figure S4 demonstrate that $\mathrm{Pb}$ atoms are absent at the cavity region of the hollow particle, while $\mathrm{Cd}$ atoms are evenly distributed throughout the whole particle, suggesting the formation of a homogenous $\mathrm{CdS}$ shell around the cavity. Note that the shell thickness could be precisely tuned by varying the reaction temperature, from 2.7 nm at $180{ }^{\circ} \mathrm{C}$ to $2.4 \mathrm{~nm}$ at $160{ }^{\circ} \mathrm{C}$ and then $2 \mathrm{~nm}$ at $130{ }^{\circ} \mathrm{C}$ (Figure S5).

For comparison, single-reaction or two-step synthesis generates products distinct from the QMNBs prepared via the synergic reactions. Firstly, PbS/CdS core/shell NCs (CSNCs) were produced by applying only cation exchange in a mixed solvent ( $9 \mathrm{~mL}$ of $\mathrm{OA}$ and $9 \mathrm{~mL}$ of ODE) under $\mathrm{N}_{2}$ flow for $10 \mathrm{~min}$ at $250{ }^{\circ} \mathrm{C}$, with $\mathrm{PbS}$ cores showing atomically sharp $\{110\}$ edges (Figures $3 \mathrm{a}$ and $3 \mathrm{~b}$ ). The ab-initio calculations indicate that the $\{111\} \mathrm{PbS} /\{111\} \mathrm{CdS}$ and $\{110\} \mathrm{PbS} /\{110\} \mathrm{CdS}$ interfaces are more stable than the $\{100\} \mathrm{PbS} /\{100\} \mathrm{CdS}$ one (see Figure $3 \mathrm{c}$ and Table S1), suggesting that cation exchange proceeds anisotropically. Secondly, PbS NCs shrank into quasi-spheres after being solely etched in OA saturated with air (Figure S6), while they remained intact after being heated in OA with $\mathrm{N}_{2}$ as protective gas (Figure S7). This indicated that the oxygen in air rather than OA was the actual etching agent. This phenomenon was observed in other studies, in which $\mathrm{PbS}$ NPs were oxidized into $\mathrm{PbO}$ and $\mathrm{PbSO}_{4}$ by air and then dissolved in solution ${ }^{43,44}$. [not clear what you meant!] Thirdly, PbS/CdS CSNCs obtained via cation exchange in $\mathrm{OA}$ under $\mathrm{N}_{2}$ flow for $35 \mathrm{~min}$ at $180^{\circ} \mathrm{C}$, which also have sharp $\{110\}$ edges on $\mathrm{PbS}$ cores (Figure S8a), were chemically etched into irregularly-shaped CdS fragments (see Supporting Information and Figure S8b for details). This demonstrated that the stepwise synthesis could not produce high-quality CdS QMNBs.
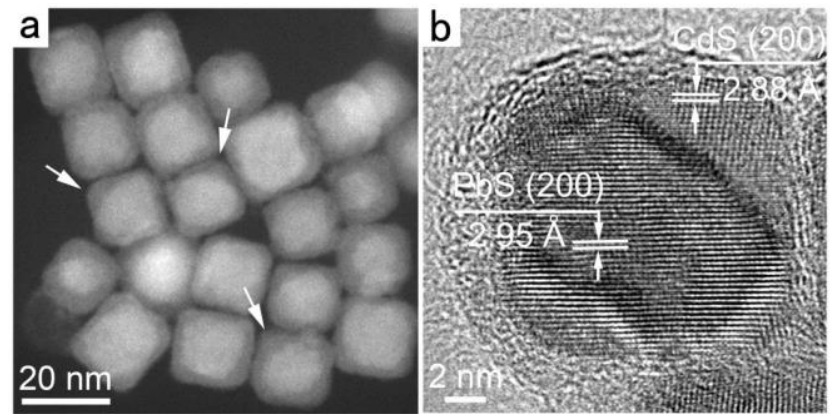

C

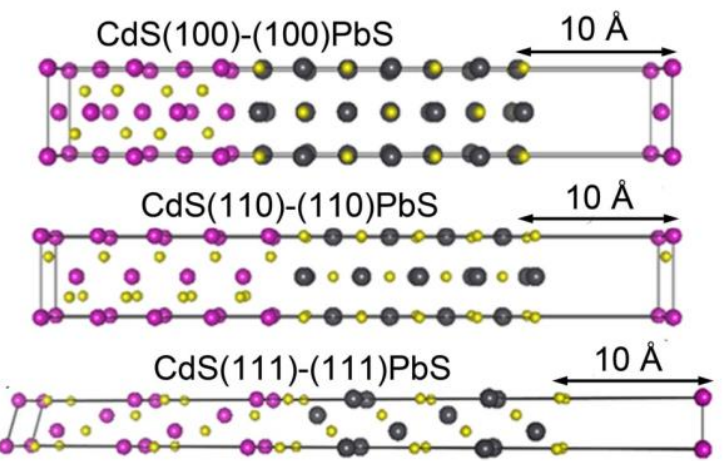

Figure 3. Products of component reactions and the calculation of interface energies. (a) and (b) are high-angle-annulardark-field scanning transmission electron microscopy (HAADF-STEM) and HRTEM images of PbS/CdS core/shell nanostructures obtained via cation exchange alone, respectively. The white arrows in (a) indicate the CdS areas. (c) Side views of three different $\mathrm{CdS} / \mathrm{PbS}$ interfaces: (100)/(100), $(110) /(110)$ and $(111) /(111)$, with pink, gray and yellow balls representing $\mathrm{Cd}, \mathrm{Pb}$ and $\mathrm{S}$ atoms, respectively. A vacuum layer with a thickness of $10 \AA$ was added during calculations to avoid the interactions between the $\mathrm{PbS}$ surface and the opposite $\mathrm{CdS}$ surface atoms.

To elucidate the mechanisms of the CdS QMNB formation, we systematically investigated intermediate products using HRTEM (Figures 4a-4f). The PdS templates exhibited high crystallinity (Figure 4a). As the reactions proceeded, first cation exchange started at the NC vertexes and edges (Figure 4b). Afterwards, small jagged cavities appeared on the $\{100\}$ surfaces of PbS NCs (Figure 4c). The cavities then expanded into the $\mathrm{PbS} \mathrm{NCs}$ while the transformed $\mathrm{CdS}$ shells remained intact (Figure 4d). Interestingly, the cation exchange did not occur to a large extent on the front wall of the freshly formed cavity (Figures $4 \mathrm{~d}$ and $4 \mathrm{e}$ ). As a result, the expansion of the cavity guided cation exchange along the NC inner surface and led to the formation of a uniform CdS shell (Figures $4 \mathrm{~d}$ and $4 \mathrm{e}$, see discussion below). Finally, the PbS core was etched out and a QMNB was obtained (Figure 4f). 


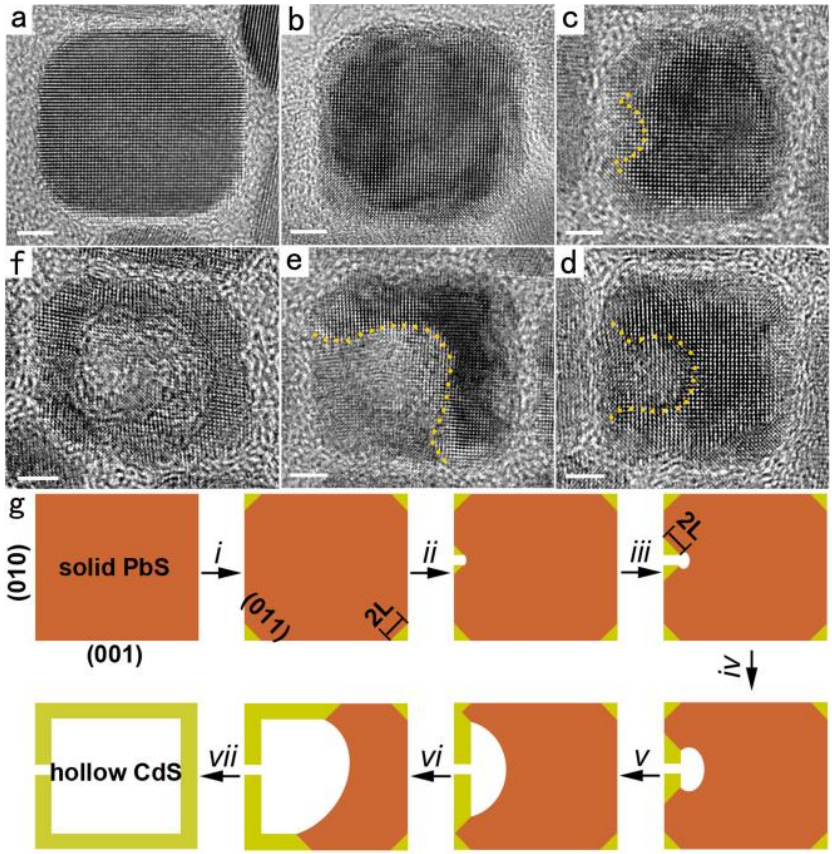

Figure 4. Structural evolution from $\mathrm{PbS}$ nanocubes to $\mathrm{CdS}$ QMNBs as synergic reactions proceed. (a)-(f) HRTEM images of products representing different stages of transformation from $\mathrm{PbS}$ NCs to hollow CdS nanoboxes. The scale bar is $3 \mathrm{~nm}$. (g) Schematic cross sectional profiles for the evolution of a $\mathrm{PbS} \mathrm{NC}$ to a $\mathrm{CdS}$ nanobox as viewed along the $\langle 100\rangle$ direction.

Based on the above HRTEM observations, the transition from $\mathrm{PbS}$ NCs to $\mathrm{CdS}$ QMNBs can be schematically illustrated, as shown in Figure 4g: (i) At the very beginning, cation exchange takes place very rapidly and proceeds in an anisotropic fashion $^{45,46}$. Since the NC vertexes and edges along the <111> and $\langle 110\rangle$ directions are more active compared with the stable $\{100\}$ planes, cation exchange preferably takes place in these regions first, and then saturates when a formed $\mathrm{CdS}$ layer reaches its equilibrium thickness, which is temperature-dependent and related to the interdiffusion free path (L) of cations on the $\{111\}$ planes $^{46}$. (ii) As the processes proceed, chemical etching attacks the exposed $\{100\}$ surfaces of PbS NCs, where it is more likely to succeed at a defect site, first producing a pinhole. In turn, the hole edges become active sites and can be exchanged with $\mathrm{Cd}^{2+}$ cations. Subsequently, cation exchange proceeds rapidly and stops as a $\mathrm{CdS}$ layer reaches its threshold thickness. (iii) As time elapses, chemical etching proceeds inwards and laterally, gradually expanding the hole into a cavity. Meanwhile, cation exchange is prohibited at the spherical front wall of the cavity, because its shape, unlike the vertexes and edges, is not favorable for cation diffusion. On the other hand, the spherical frontier keeps dissolving and moving on, thus creating very unfavorable conditions for the nucleation and steady growth of CdS on its surface. Being intrinsically a nucleationgrowth process ${ }^{46}$, the cation exchange cannot proceed on such an "unstable" surface. In contrast, the NC wall area undergoes severe cation exchange as now the CdS around the hole acts as stable nuclei and the cation interdiffusion can proceed easily there, from both outside and inside. As a result, the CdS area grows with the expansion of the cavity. Particularly, the thick- ness of the CdS layer stays constant in accordance with the interdiffusion free path length of cations. (iv-vii) Next, chemical etching continues to hollow out the $\mathrm{PbS} \mathrm{NC}$, and the exchanged $\mathrm{CdS}$ walls advance along the $\mathrm{PbS} \mathrm{NC}$ contour, guiding cation exchange to a complete, uniform, and single-crystal CdS shell. Eventually, this results in the formation of a CdS QMNB. For $\mathrm{CdS}$ material, the zinc-blende phase is more stable than the wurtzite phase when nanoparticle size is smaller than $6 \mathrm{~nm}^{47}$. Therefore, the zinc-blende phase is stabilized in the CdS nanoboxes with the wall thickness of $2.5 \mathrm{~nm}$.

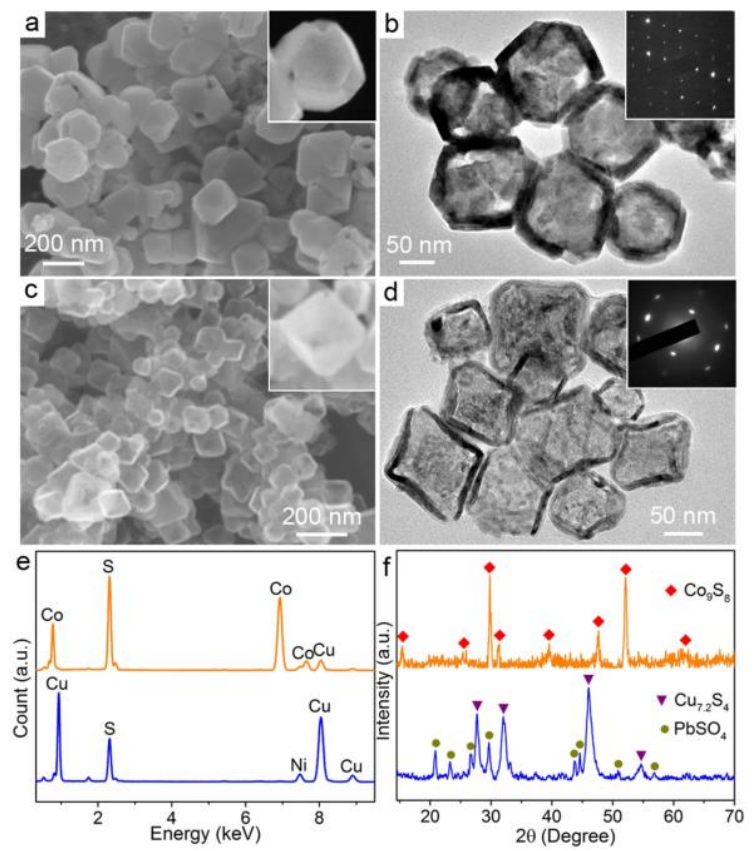

Figure 5. Single-crystalline hollow $\mathrm{Co}_{9} \mathrm{~S}_{8}$ and $\mathrm{Cu}_{7.2} \mathrm{~S}_{4}$ nanooctahedrons synthesized via the reaction of $\mathrm{PbS}$ nanooctahedrons with $\mathrm{OA}$-dissolved $\mathrm{Co}(\mathrm{Ac})_{2}$ and $\mathrm{Cu}(\mathrm{Ac})_{2}$, respectively. (a) SEM and (b) TEM images of $\mathrm{Co}_{9} \mathrm{~S}_{8}$; (c) SEM and (d) TEM images of $\mathrm{Cu}_{7.2} \mathrm{~S}_{4}$; (e) EDS spectra and (f) XRD patterns of the products presented in panels (a), (b) and (c), (d). The insets in (b) and (d) are SAED patterns of one particle. In EDS spectra of panel (e), $\mathrm{Cu}$ signals in $\mathrm{Co}_{9} \mathrm{~S}_{8}$ and $\mathrm{Ni}$ signals in $\mathrm{Cu}_{7.2} \mathrm{~S}_{4}$ originate from $\mathrm{Cu}$ and $\mathrm{Ni}$ grids, respectively.

To extend the synergic reaction synthesis approach, we then employed $\mathrm{Co}^{2+}$ and $\mathrm{Cu}^{2+}$ cations instead of $\mathrm{Cd}^{2+}$ to attack $\mathrm{PbS}$ octahedron templates. It was found that $\mathrm{Co}_{9} \mathrm{~S}_{8}$ and $\mathrm{Cu}_{7.2} \mathrm{~S}_{4}$ single-crystalline hollow nanostructures were respectively obtained (Figure 5). SEM and TEM images (Figures 5a-5d) demonstrate that both products are hollow nanooctahedrons with single-crystal shells, which is confirmed by SEAD patterns in the insets of Figures $5 \mathrm{~b}$ and 5d. EDS (Figure 5e) and XRD (Figure 5f) results reveal that the rock-salt $\mathrm{PbS}$ templates were consumed completely, and the obtained hollow nanooctahedrons are rock-salt $\mathrm{Co}_{9} \mathrm{~S}_{8}$ and $\mathrm{Cu}_{7.2} \mathrm{~S}_{4}$. 

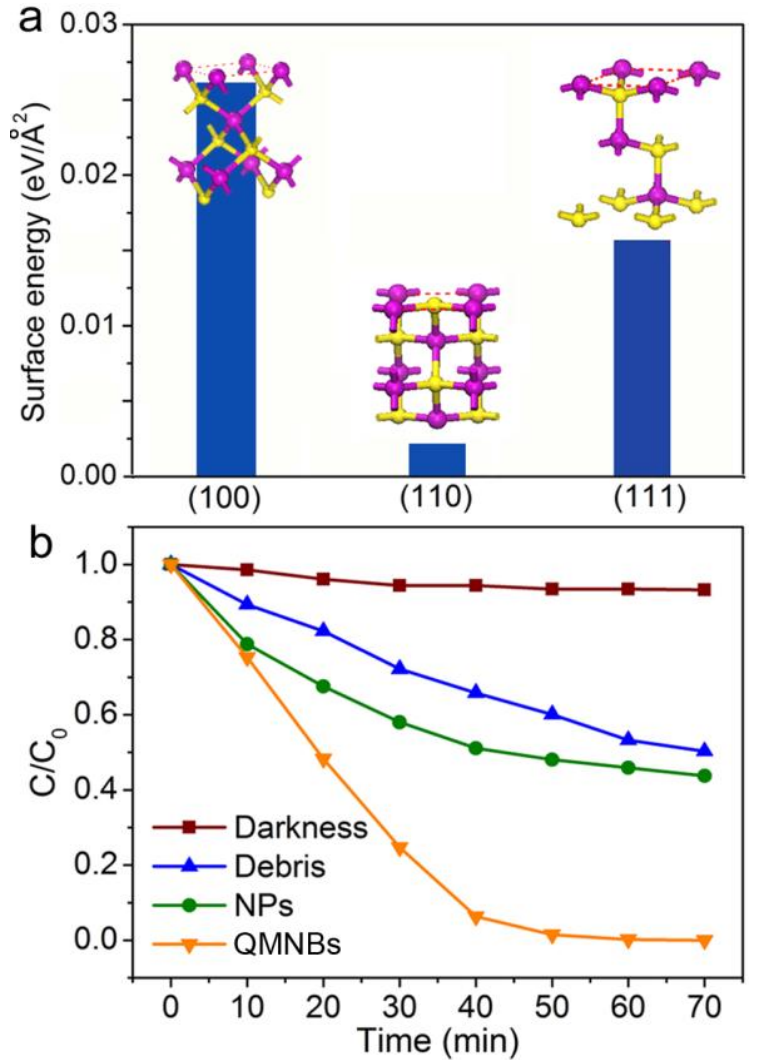

Figure 6. Surface energy and photocatalytic activity of CdS QMNBs. (a) Surface energies for three facets of zinc-blende CdS. (b) Photocatalytic decay of MB by CdS nanoboxes, NPs and debris. The intensity change of MB absorption at $663 \mathrm{~nm}$ was recorded at different light-irradiation times. All samples were treated with EDA prior to mixing with MB solution and measured under the same conditions.

The prepared CdS QMNBs exhibit unique $\{100\}$ facets and a large specific surface area $\left(63 \mathrm{~m}^{2} \mathrm{~g}^{-1}\right)$, which is of great benefit for photocatalytic properties. First, density function theory (DFT) calculations were made to determine the surface energy of CdS QMNBs. Among the low-index planes $\{100\},\{110\}$ and $\{111\}$ of the zinc-blende CdS phase, the $\{100\}$ planes own the highest surface energy $\left(0.02657 \mathrm{eV} / \AA^{2}\right)$, even an order of magnitude larger than those of $\{110\}\left(0.00255 \mathrm{eV} / \AA^{2}\right)$ and $\{111\}$ $\left(0.01610 \mathrm{eV} / \AA^{2}\right)$ planes (Figure 6a). Therefore, it is expected that the CdS QMNBs with exposed $\{100\}$ facets would possess a high dye adsorption capability and photocatalytic activity ${ }^{48-50}$. Accordingly, we tested the photocatalytic performance of CdS QMNBs after OA was stripped from their surface (Figure S9), by adopting the photodegradation of methylene blue (MB) as the probe reaction ${ }^{51}$. Two other samples, CdS NPs prepared via a combination reaction (see Supporting Information and Figure S10 for details) and the CdS debris synthesized by the previously mentioned stepwise method (see Supporting Information and Figure S8b for details), were used as references. As shown in Figure 6b, almost no any degradation of MB occurred with CdS QMNBs in darkness. Under light irradiation, the QMNBs exhibited superior photodegradation efficiency compared to the CdS NPs with the same quantity but a larger specific surface area $\left(77.09 \mathrm{~m}^{2} \mathrm{~g}^{-1}\right)$. Since the three samples have identical composition and crystal structure but different exposed surfaces, it can be concluded that the excellent photocatalytic properties of the QMNBs are attributed to their high-energy $\{100\}$ facets which provide highly-active sites for the adsorption and degradation of organic molecules.

\section{CONCLUSIONS}

In summary, we have demonstrated that the synergic reaction synthesis strategy combining the merits of cation exchange and chemical etching can generate diverse hollow nanostructures. More importantly, we found that the obtained $\mathrm{CdS}$ QMNBs own a metastable phase, exposed high-energy facets, the smallest size reported so far, and rather large specific surface area, which jointly provide promising and exceptional catalytic activities. Our results demonstrate the high potential of synergic reactions in engineering of complex nanostructures with high quality and superior properties.

\section{ASSOCIATED CONTENT}

\section{Supporting Information}

Detailed synthetic procedures, characterization methods, calculation and additional experimental results. This material is available free of charge via the Internet at http://pubs.acs.org.

\section{AUTHOR INFORMATION}

\section{Corresponding Author}

* Address correspondence to xwdu@tju.edu.cn and s.qiao@adelaide.edu.au.

\section{Notes}

The authors declare no competing financial interest.

\section{ACKNOWLEDGMENT}

This work was supported by the National Basic Research Program of China (2014CB931703), the Natural Science Foundation of China (Nos. 51171127, 51102176, and 51271129). Research carried out in part at the Center for Functional Nanomaterials, Brookhaven National Laboratory, which is supported by the U.S. Department of Energy, Office of Basic Energy Sciences, under Contract No. DE-SC0012704.

\section{DEFERENCES}

(1) An, K.; Hyeon, T. Nano Today 2009, 4, 359.

(2)Wang, L.; Nemoto, Y.; Yamauchi, Y. J. Am. Chem. Soc. 2011, 133, 9674.

(3) Zhang, Q.; Wang, W.; Goebl, J.; Yin, Y. Nano Today 2009, 4, 494.

(4) Lou, X. W.; Archer, L. A.; Yang, Z. Adv. Mater. 2008, $20,3987$.

(5) Wang, Z.; Luan, D.; Boey, F. Y. C.; Lou, X. W. J. Am. Chem. Soc. 2011, 133, 4738.

(6) Nai, J. W.; Tian, Y.; Guan, X.; Guo, L. J. Am. Chem. Soc. 2013, 135,16082

(7) Qin, Z. H.; Sun, H. Y.; Jiang, Z.; Jiao, X. L.; Chen, D. R. CrystEngComm 2013, 15, 897.

(8) Chen, C.; Kang, Y. J.; Huo, Z. Y.; Zhu, Z. W.; Huang, W. Y.; Xin, H. L. L.; Snyder, J. D.; Li, D. G.; Herron, J. A.; Mavrikakis, M.; Chi, M. F.; More, K. L.; Li, Y. D.; Markovic, N. M.; Somorjai, G. A.; Yang, P. D.; Stamenkovic, V. R. Science 2014, 343, 1339.

(9) Afanasiev, P. J. Phys. Chem. C 2012, 116, 2371.

(10) Gonzalez, E.; Arbiol, J.; Puntes, V. F. Science 2011, 334, 1377. 
(11) An, K.; Kwon, S. G.; Park, M.; Bin Na, H.; Baik, S. I.; Yu, J. H.; Kim, D.; Son, J. S.; Kim, Y. W.; Song, I. C.; Moon, W. K.; Park, H. M.; Hyeon, T. Nano Lett. 2008, 8, 4252.

(12) Zhang, L.; Zhou, L.; Wu, H. B.; Xu, R.; Lou, X. W.Angew. Chem. Int. Ed. 2012, 51, 7267.

(13) Tian, L.; Yang, X.; Lu, P.; Williams, I. D.; Wang, C.; Ou, S.; Liang, C.; Wu, M. Inorg. Chem. 2008, 47, 5522.

(14)Yang, H. G.; Zeng, H. C. J. Phys. Chem. B 2004, 108, 3492.

(15) Camargo, P. H. C.; Lee, Y. H.; Jeong, U.; Zou, Z. Q.; Xia, Y. N. Langmuir 2007, 23, 2985.

(16) Rivest, J. B.; Jain, P. K. Chem. Soc. Rev. 2013, 42, 89.

(17) Kim, M. R.; Jang, D. J. Chem. Commun. 2008, 5218.

(18) Zhu, H. T.; Wang, J. X.; Wu, D. X. Inorg. Chem., 2009, 48, 7099.

(19) Moon, G. D.; Ko, S.; Min, Y.; Zeng, J.; Xia, Y.; Jeong, U. Nano Today 2011, 6, 186.

(20) Zhao, W.; Zhang, C.; Geng, F.; Zhuo, S.; Zhang, B. ACS Nano 2014, 8,10909 .

(21) Apte, S. K.; Garaje, S. N.; Mane, G. P.; Vinu, A.; Naik, S. D.; Amalnerkar, D. P.; Kale, B. B. Small 2011, 7, 957.

(22) Jang, J. S.; Joshi, U. A.; Lee, J. S. J. Phys. Chem. C 2007, 111, 13280 .

(23)Tong, H.; Umezawa, N.; Ye, J.; Ohno, T. Energy Environ. Sci. 2011, 4, 1684.

(24) Yin, P.-F.; Ling, T.; Lu, Y.-R.; Xu, Z.-W.; Qiao, S.-Z.; Du, X.-W. Adv. Mater. 2015, 27, 740 .

(25) Li, H.; Wang, X.; Xu, J.; Zhang, Q.; Bando, Y.; Golberg, D.; Ma, Y.; Zhai, T. Adv. Mater. 2013, 25, 3017.

(26) Kwak, W.-C.; Han, S.-H.; Kim, T. G.; Sung, Y.-M. Cryst. Growth Des. 2010, 10, 5297.

(27) Xu, D.; Liu, Z. P.; Liang, J. B.; Qian, Y. T. J. Phys. Chem. B 2005 , 109,14344

(28) Zhai, T.; Fang, X.; Bando, Y.; Liao, Q.; Xu, X.; Zeng, H.; Ma, Y.; Yao, J.; Golberg, D. ACS Nano 2009, 3, 949.

(29) Panda, A. B.; Glaspell, G.; El-Shall, M. S. J. Am. Chem. Soc. 2006, $128,2790$.

(30) Bao, N. Z.; Shen, L. M.; Takata, T.; Domen, K. Chem. Mater. 2008, 20,110 .

(31) Huang, Y. Y.; Sun, F. Q.; Wang, H. J.; He, Y.; Li, L. S.; Huang, Z. X.; Wu, Q. S.; Yu, J. C. J. Mater. Chem. 2009, 19, 6901.
(32) Fan, Z.; Razavi, H.; Do, J.-w.; Moriwaki, A.; Ergen, O.; Chueh, Y.-L.; Leu, P. W.; Ho, J. C.; Takahashi, T.; Reichertz, L. A.; Neale, S.; Yu, K.; Wu, M.; Ager, J. W.; Javey, A. Nat. Mater. 2009, 8, 648.

(33) Ma, R. M.; Dai, L.; Huo, H. B.; Yang, W. Q.; Qin, G. G.; Tan, P. H.; Huang, C. H.;Zheng, J. Appl. Phys. Lett. 2006, 89, 203120.

(34) Zhang, N.; Yang, M. Q.; Tang, Z. R.; Xu, Y. J. ACS Nano 2014, 8,623 .

(35) Hu, Y.; Liu, Y.; Qian, H. S.; Li, Z. Q.; Chen, J. F. Langmuir 2010, 26, 18570.

(36) Cao, Y. C.; Wang, J. H. J. Am. Chem. Soc. 2004, 126, 14336.

(37) Tong, H.; Ouyang, S. X.; Bi, Y. P.; Umezawa, N.; Oshikiri, M.; Ye, J. H. Adv. Mater. 2012, 24, 229.

(38) Yang, H. G.; Sun, C. H.; Qiao, S. Z.; Zou, J.; Liu, G.; Smith, S. C.; Cheng, H. M.; Lu, G. Q. Nature 2008, 453, 638.

(39) Joo, J.; Na, H. B.; Yu, T.; Yu, J. H.; Kim, Y. W.; Wu, F. X.; Zhang, J. Z.; Hyeon, T. J. Am. Chem. Soc. 2003, 125, 11100.

(40) Perdew, J. P.; Burke, K.; Ernzerhof, M. Phys. Rev. Lett. 1996, 77, 3865 .

(41) Rodic, D.; Spasojevic, V.; Bajorek, A.; Onnerud, P. J. Magn. Magn. Mater. 1996, 152, 159

(42) Nag, A.; Hazarika, A.; Shanavas, K. V.; Sharma, S. M.; Dasgupta, I.; Sarma, D. D. J. Phys. Chem. Lett. 2011, 2, 706.

(43) Sadovnikov, S. I.; Kozhevnikova, N. S.; Rempel, A. A. Russ. J. Inorg. Chem. 2011, 56, 1864.

(44) Sykora, M.; Koposov, A. Y.; McGuire, J. A.; Schulze, R. K.; Tretiak, O.; Pietryga, J. M.; Klimov, V. I. ACS Nano 2010, 4, 2021.

(45) Chan, E. M.; Marcus, M. A.; Fakra, S.; ElNaggar, M.; Mathies, R. A.; Alivisatos, A. P. J. Phys. Chem. A 2007, 111, 12210.

(46) Casavola, M.; van Huis, M. A.; Bals, S.; Lambert, K.; Hens, Z.; Vanmaekelbergh, D. Chem. Mater. 2012, 24, 294.

(47) Banerjee, R.; Jayakrishnan, R.; Ayyub, P. J. Phys.: Condens. Matter 2000, 12, 10647.

(48) Gong, X. Q.; Selloni,A. J. Phys. Chem. B 2005, 109, 19560.

(49) Selloni, A. Nat. Mater.2008, 7, 613.

(50) Pan, J.; Liu, G.; Lu, G. Q.; Cheng, H. M. Angew. Chem. Int. Ed. 2011, 50, 2133.

(51) Zhang, H.; Lv, X. J.; Li, Y. M.; Wang, Y.; Li, J. H. ACS Nano 2010, 4, 380 . 
SYNOPSIS TOC
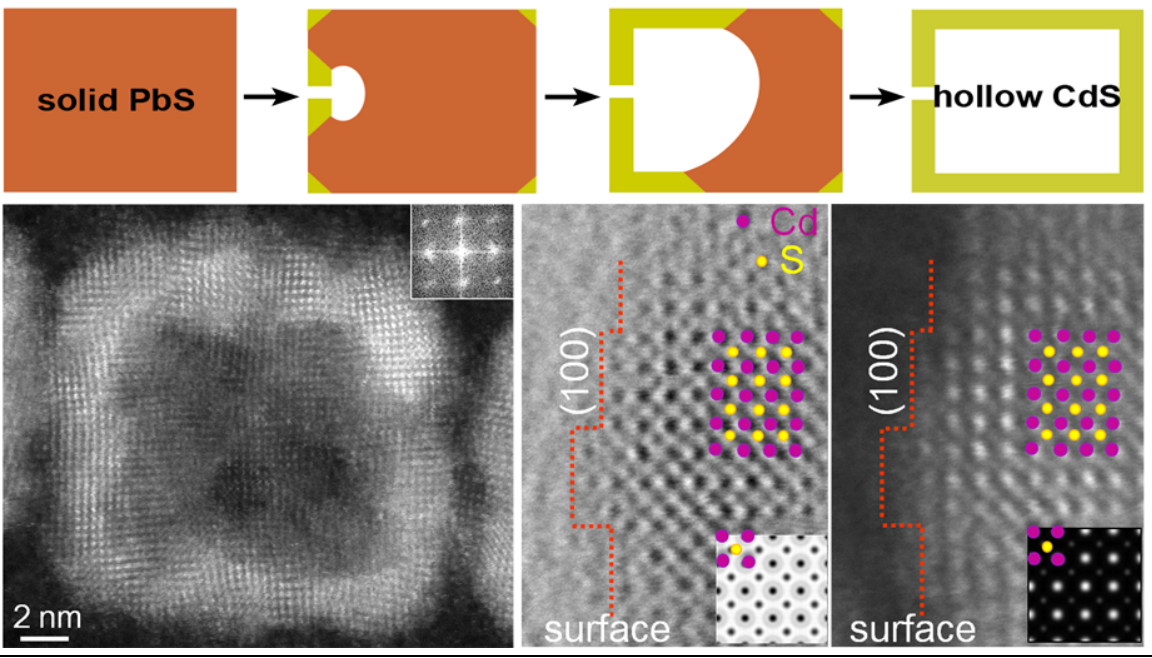